\title{
5 Research Square \\ Three-dimensional ultrasonography study of female defecation disorder caused by changes of the puborectalis muscle
}

Hui Zhang

Weifang Medical University

Kailun Liu

rizhao people's hospital

Zhimin Wang ( $\sim$ jnwzm@126.com )

Fanjuan Kong

rizhao hospital of traditional chinese medicine

\section{Rongqing Gao}

Weifang Medical University

\section{Lu Chen}

Weifang Medical University

Yuantao Li

Shandong Qianfoshan Hospital

Xue Pang

Shandong Qianfoshan Hospital

\section{Research article}

Keywords: three-dimensional ultrasonography, pelvic floor structure, puborectalis angle, puborectalis thickness, pelvic floor dyssynergia-type constipation

Posted Date: July 25th, 2019

DOl: https://doi.org/10.21203/rs.2.11931/v1

License: (c) (1) This work is licensed under a Creative Commons Attribution 4.0 International License.

Read Full License 


\section{Abstract}

Objective To investigate the correlation between pelvic floor dyssynergia-type constipation and the puborectalis muscle using three-dimensional ultrasonography. Methods Eighty-seven patients with pelvic floor dyssynergia-type constipation (observation group) and 87 healthy volunteers (control group) were enrolled in this study. Three-dimensional ultrasonography of the pelvic floor was performed on patients and healthy volunteers in a lithotomy position at rest and during straining and squeezing. The threedimensional reconstructed sonogram was analysed to determine the puborectalis angle and puborectalis thickness at the 6 o'clock position (patients in a lithotomy position). Results (1) The puborectalis angles of the observation and control groups were not significantly different at rest [(85.664 \pm 1.926$)^{\circ},(85.813 \pm$ $\left.1.500)^{\circ}\right]$ and during squeezing $\left[(87.478 \pm 2.125)^{\circ},(86.960 \pm 1.751)^{\circ}\right](P>0.05)$ but were significantly different when straining $\left[(80.389 \pm 2.268)^{\circ},(94.382 \pm 1.540)^{\circ}\right](P<0.05)$. The difference in the puborectalis angles of the two groups between rest and straining $\left[(5.275 \pm 1.236)^{\circ},(-8.569 \pm 1.209)^{\circ}\right]$ was statistically significant $(P<0.05)$. (2) The puborectalis thickness of the observation and control groups was not significantly different at rest $([3.994 \pm 0.128) \mathrm{mm},(3.983 \pm 0.091) \mathrm{mm}]$ and during anal squeezing $[(4.082 \pm 0.154) \mathrm{mm},(4.126 \pm 0.113) \mathrm{mm}](P>0.05)$ but was significantly different when straining $[(4.630 \pm 0.199) \mathrm{mm},(4.296 \pm 0.121) \mathrm{mm}](P<0.05)$. The differences in the puborectalis thickness at rest and during straining in the observation and control groups were $(-0.636 \pm 0.217) \mathrm{mm}$ and $(-0.316 \pm 0.089) \mathrm{mm}$, respectively, resulting in a statistically significant difference between the two groups $(P<0.05)$. Conclusion The puborectalis angle in patients with pelvic floor dyssynergia-type constipation was smaller than that in healthy volunteers during straining. The puborectalis thickness at the 6 o'clock position with the patient in a lithotomy position in patients with pelvic floor dyssynergia-type constipation was larger than that in healthy volunteers. Moreover, larger differences between rest and straining are associated with a more severe degree of constipation. Project funding Natural Science Foundation of Shandong (ZR2012HM086)

\section{Introduction}

Chronic constipation is a common disease and is usually divided into primary and secondary constipation, with a prevalence rate of $10-15 \%{ }^{[1]}$. It can significantly affect the quality of life of patients. Primary constipation is divided into outlet obstruction constipation and slow transit constipation ${ }^{[2]}$. A recent study reported that the occurrence of primary chronic constipation is correlated with changes in pelvic floor muscles ${ }^{[3]}$. The development and progress of modern imaging technology has led to increased study of pelvic floor dysfunction diseases, which has drawn increasing attention to "pelvic floor dyssynergia-type constipation" [4]. Defecography can only provide an evaluation of the contour, course and relationship with the surrounding organs and the motility function of the large intestine ${ }^{[5]}$. Magnetic resonance imaging (MRI) defecography (MRId) can provide morphological and functional images of the pelvic floor structures with good contrast with soft tissue, but patients with metal pacemakers and intrauterine contraceptive devices (IUDs) are contraindicated to this examination ${ }^{[6]}$. Benacerraf et al. ${ }^{[7]}$ stated that three-dimensional ultrasonography imaging is the first choice for the study of the uterus, 
endometriosis and ovaries in women. Three-dimensional ultrasonography can simultaneously display three cross-sections (sagittal, transverse and coronal sections) that are perpendicular to one another to obtain a complete three-dimensional image of the pelvic hiatus. The reconstructed three-dimensional images can be used to evaluate the intactness, thickness and volume of the levator ani of the patient ${ }^{[8]}$. Rotenberg et al. ${ }^{[9]}$ suggested that transvaginal ultrasonography has certain limitations for pelvic floor examination because the ultrasound probe can cause pelvic floor dilation, deformation and muscle stretching while being placed in the vagina. This study used three-dimensional perineal ultrasonography to observe the relationship between pelvic floor dyssynergia-type constipation and changes in the puborectalis angle or puborectalis thickness at the pelvic floor at rest and during straining and squeezing.

\section{Materials And Methods}

\section{Research subjects}

A total of 87 female constipation patients who visited the anorectal clinic of the Qianfoshan Hospital affiliated to Shandong University or were hospitalised from June 2016 to October 2017 were randomly selected. Their age, height and body mass index were (50.75 \pm 11.41$)$ years (range: 24 to 73 years), $(159.63 \pm 5.91) \mathrm{cm}$ and $(21.42 \pm 1.59)$, respectively. The patients had no history of pelvic surgery, pelvic mass or chronic cough but had undergone one virginal delivery. All patients whose defecography and colonic transit time test results were normal met the Rome III diagnostic criteria for functional constipation.

A total of 87 women who presented in the outpatient clinic of the Department of Obstetrics and Gynaecology during same time period and were diagnosed with irregular menstruation and vaginitis were randomly selected as the control subjects. Their age, height and body mass index were $(51.19 \pm 9.67)$ years (range: 28 to 69 years), $(160.16 \pm 6.00) \mathrm{cm}$ and $(21.75 \pm 1.63)$, respectively. The patients had no history of pelvic surgery, pelvic mass or chronic cough but had undergone one vaginal delivery. This study was approved by the Ethics Committee of our hospital, and all patients signed an informed consent form.

\section{Instruments and methods}

A colour Doppler ultrasonographic diagnostic scanner (Pro FOCUS 2202, BK Medical ApS) was used in this study. The 3600 rotary mechanical transducer probes were used with the following settings: frequency, 2-6 MHz; adjustable centre frequency, level 4; detection angle, $360^{\circ}$ and depth, $290 \mathrm{~mm}$.

A three-dimensional ultrasonography examination of the pelvic floor was performed by the same experienced and professionally trained operator who was blinded to the study data. The subjects were trained with a standardised pelvic floor biofeedback therapy device (usually trained 3 to 5 times) to perform anal straining and squeezing. The subjects were lying down in a lithotomy position after emptying their bladder. A transducer probe coated with an appropriate amount of ultrasonic coupling 
agent was covered by a condom, and the air inside the condom was evacuated before the probe was used to measure the puborectalis angle and thickness at the 6 o'clock position with the patient in a lithotomy position. An appropriate amount of ultrasonic coupling agent was applied into the vagina so that the transducer probe could better contact with the wall of vagina to reduce the artefact interference. The static three-dimensional acquisition system started to acquire the three-dimensional image of the pelvic floor at rest. Volume scanning was performed from one edge of the target to the other edge, and the images were adjusted to obtain mutually perpendicular sagittal, coronal and transverse sections. After obtaining satisfactory volume data, the images were frozen and stored. Similarly, a threedimensional image of the pelvic floor was acquired at rest and during straining and squeezing. After completion of image collection, the three-dimensional images were reconstructed. The measured parameters are as follows: $\triangle$ Puborectalis angle: the puborectalis angle was defined as the angle between the projected lines from the internal edge of the junction (6 o'clock position) of the left and right aspects of the puborectalis muscle posterior to the rectum to the 3 and 9 o'clock positions of the rectum, respectively, with the patient in a lithotomy position (Figure 1); $\otimes$ The puborectalis thickness at the 6 o'clock position of the rectum (with the patient in a lithotomy position): the puborectalis thickness was measured in the sagittal plane.

\section{Statistical Analysis}

SPSS 21.0 statistical software was used for the statistical analysis. The data are expressed as. Paired ttests were used to compare the measurement data, puborectalis angle and puborectalis thickness. The significance level was set at $a=0.05$; a value of $P<0.05$ was considered statistically significant.

\section{Results}

Three-dimensional ultrasonography images were obtained at rest and during straining and squeezing in the observation and control groups. These images were processed to obtain complete images of the anal hiatus of the pelvic diaphragm at the level of the lower edge of pubic symphysis and anorectal junction in the titling cross-sectional, coronal and sagittal planes. We successfully measured the related parameters in the titling cross-sectional and sagittal planes. Comparison of the puborectalis angle between the observation and control groups, the difference of the puborectalis angle between rest and straining, the puborectalis thickness at the 6 o'clock position (with the patient in a lithotomy position) and the difference in puborectalis thickness at the 6 o'clock position (with the patient in a lithotomy position) between rest and straining are shown in Tables 1 and 2 .

This study showed no significant difference in the puborectalis angle and puborectalis thickness between the observation and control groups during rest and anal squeezing $(P>0.05)$. The changes in the puborectalis angle and puborectalis muscle thickness were significantly different between the observation and control groups $(P<0.05)$. The differences in the puborectalis angle and puborectalis thickness between rest and straining between the observation and control groups were statistically 
significant $(P<0.05)$, and a greater difference was associated with an increased degree of difficulty of defecation (Figures 3 and 4).

\section{Discussion}

In recent years, the study of the relationship between pelvic floor dysfunction and functional constipation has become a focus of research ${ }^{[10]}$. In the past, pelvic floor dyssynergia-type constipation was attributed to rectal protrusion and rectal intussusception and collectively called pelvic floor dysfunction syndrome. According to clinical experience, the postoperative effect in most patients is still not satisfactory due constipation recurrence ${ }^{[11]}$, regardless of position of rectal mucosa suspension, range of tissue removal or enhanced repair of local muscle tissue. Due to the continuous development of three-dimensional ultrasonography techniques, this method has been increasingly applied to pelvic floor imaging ${ }^{[12]}$. The structures of the puborectalis muscle can be accurately assessed by three-dimensional perineal ultrasonography, which is convenient and non-invasive. The anatomical structures can be measured in the pubic symphysis, bilateral pubic ramus and medial and posterior edges of the puborectalis muscle $[13,14]$. Based on many clinical observations and data measurements, this study has shown that the puborectalis thickness at the 6 o'clock position (with patients in a lithotomy position) is an important factor. This study suggests that changes in the puborectalis angle and thickness may be important contributors to pelvic floor dyssynergia-type constipation.

The puborectalis muscle is a striated muscle comprised of strip-shaped muscular fibres. The puborectalis muscle originates from the lower part of the pubic symphysis and is adjacent to the pubis. It extends posterior-inferiorly around the vagina or the prostate and merges to form an intact muscle behind the anorectal junction to retract the anorectal junction anteriorly to form an anorectal angle. The puborectalis muscle is a powerful " $U$ "-shaped "sling" and the most powerful part of the levator ani muscle ${ }^{[15,16]}$. The puborectalis pubococcygeus, and iliococcygeal muscles form the majority of the levator plate ${ }^{[17]}$. The puborectalis muscle is more important than the pubococcygeus muscle and the iliococcygeal muscle. Previously, the anorectal angle and levator plate were considered to be good parameters to evaluate the pelvic floor structure ${ }^{[18]}$, which plays an important role in defecation. However, this study suggested that the puborectalis muscle plays a decisive role in the evaluation of the pelvic floor structure. In normal defecation, the puborectalis muscle relaxes (i.e., increased puborectalis thickness), and the anorectal junction moves to the dorsal side in the sagittal plane to enlarge the anorectal angle and complete defecation. In contrast, in patients with pelvic floor dyssynergia-type constipation, the muscle movements are in the opposite direction ${ }^{[19]}$. In the 68 patients in the observation group, three-dimensional ultrasonography showed that the puborectalis angle was larger at rest than during straining. In the 67 patients in the control group, the puborectalis angle was smaller at rest than during straining, and only one patient had a larger puborectalis angle at rest than during straining. The difference in the puborectalis thickness between rest and straining was smaller in the observation group than in the control group. Although the puborectalis angle is larger at rest than during straining, the puborectalis thickness at the 6 o'clock position is smaller at rest than during straining. 
In females, the pelvic floor hiatus may be injured during vaginal delivery ${ }^{[20]}$. In the observation group, three-dimensional ultrasonography showed a hypoechoic area in the right aspect of the puborectalis muscle in multiparas, which may be the consequence of changes or avulsion in pelvic floor tissue caused by obstetric procedures. Studies have shown that nearly half of women with a history of vaginal delivery have anatomical changes in the puborectalis muscle ${ }^{[21,22]}$. A study by Serdar et al. ${ }^{[23]}$ found that maternal overwork, oversized head circumference and an overweight of foetus can lead to puborectalis muscle injury, which causes changes in the pelvic hiatus, deformation of muscular fibres and muscle tone decline. In the observation group, three-dimensional ultrasonography showed that the puborectalis muscle is discontinuous in some patients. This discontinuation is more common in the junction of the pubic symphysis and the right aspect of the puborectalis muscle ${ }^{[24]}$. Thus, the left and right sides of the anorectal junction were unevenly affected during defecation. The left aspect of the puborectalis muscle produces excessive retraction at the anorectal junction. This prevents the puborectalis angle from expanding, and the puborectalis muscle is unable to be completely relaxed. As a consequence, varying degrees of pelvic floor dyssynergia-type constipation may occur ${ }^{[10]}$. This study suggests that pregnancy or changes in puborectalis muscle continuity can cause changes in the puborectalis angle and puborectalis thickness at the 6 o'clock position when patients are in a lithotomy position. Moreover, ageing is associated with degeneration and loosening of the pelvic floor support structures. Thus, the elasticity, extensibility, excitability and conductivity of the puborectalis muscle fibres are greatly reduced with ageing ${ }^{[17]}$. The anteroposterior diameter of the pelvic floor hiatus cannot be shortened, and the transverse diameter cannot be elongated accordingly. The differences in the puborectalis angle and the puborectalis thickness at the 6 o'clock position (with patients in a lithotomy position) between rest and straining were also increased. Follow-up of patients with constipation in this study showed that the increased differences in the puborectalis angle and the puborectalis thickness at the 6 o'clock position are associated with worsening symptoms of pelvic floor dyssynergia-type constipation (see Figures 3 and 4).

This study used three-dimensional ultrasonography to observe the changes in the shape and function of the puborectalis angle as well as changes in the puborectalis thickness at the 6 o'clock position with patients in the lithotomy position during rest, straining and squeezing. The results showed that the puborectalis angle decreased during straining of the pelvic floor structures in patients with pelvic floor dyssynergia-type constipation, and a smaller angle was associated with more serious symptoms of pelvic floor dyssynergia-type constipation. The puborectalis thickness at the 6 o'clock position is increased during straining, and the greater difference from that at rest is associated with more severe symptoms of pelvic floor dyssynergia-type constipation. Three-dimensional ultrasonography also has its limitations and is susceptible to more factors than two-dimensional ultrasonography. MRI can clearly show the pelvic floor structures.

\section{Conclusion}


The puborectalis angle in patients with pelvic floor dyssynergia-type constipation was smaller than that in healthy volunteers during straining. The puborectalis thickness at the 6 o'clock position with the patient in a lithotomy position in patients with pelvic floor dyssynergia-type constipation was larger than that in healthy volunteers. Moreover, larger differences between rest and straining are associated with a more severe degree of constipation.

\section{Abbreviations}

Magnetic resonance imaging MRI

Magnetic resonance imaging defecography MRId

intrauterine contraceptive devices IUDs

\section{Declarations}

Ethics approval and consent to participate

Consent for Publication :

Written informed consent for publication was obtained from all participants.

All of data and material are available.

All authors have no conflicts of interest.

Funding:

Natural Science Foundation of Shandong (ZR2012HM086)

Authors' contributions:

Zhimin Wang:Hosted and participated in the design and implementation of the project.

Hui Zhang and Kailun Liu:Select patients and healthy volunteers, group, measure data, collect data and write papers.

Fanjuan Kong, Rongqing Gao, Lu Chen, Yuantao Li, and Xue Pang:Perform statistical analysis data as well as assist measurement and record data.

Acknowledgement

Thanks for the cooperation of the patients; thank Professor Wang for his academic guidance; thank the teachers of the radiology department for their patient guidance; and thank the hospital leaders for their support. 


\section{References}

1. Vitton $\mathrm{V}, \mathrm{Damon} \mathrm{H}$, Benezech $\mathrm{A}$, et al. Clinical practice guidelines from the French National Society of Coloproctology in treating chronic constipation. European journal of gastroenterology \& hepatology, 2018,30(4):357-363.

2. Bharucha AE, Wald A, Enck P. Functional anorectal disorders. Gastroenterology, 2006,130(5):15101518.

3. Li R, Song Y. Relationship between levator ani and bony pelvis morphology and clinical grade of prolapse in women. Clinical anatomy, 2015,28(6):813-819.

4. Wieczorek AP, Stankiewicz A, Santoro GA, et al.Pelvic floor disorders: role of new ultrasonographictechniques[J]. World J Urol, 2011, 29(5):615-623.

5. Stellingwerf ME, Maeda $Y$, Patel $U$, et al. The role of the defaecating pouchogram in the assessment of evacuation difficulty after restorative proctocolectomy and pouch-anal anastomosis. Colorectal disease, 2016,18(8): 0292-300.

6. Lapray JF, Costa P, Delmas V. The role of medical imaging in the exploration of pelvic floor disorders. Prog Urol, 2009,19(13):953-969.

7. Benacerraf BR, Abuhamad AZ, Bromley B,et al.Consider ultrasound first for imaging the female pelvis[J].Am J Obstet Gynecol,2015,212(4):450-455.

8. Wieczorek AP, Stankiewicz A, Santoro GA, et al. Pelvic floor disorders: role of new ultrasonographic techniques. World journal of urology, 2011,29(5):615-623.

9. Rotenberg 0 , Greston WM. Three-dimensional saline infusion vaginography for sonographic assessment of the anterior vaginal wall and pelvic floor descent. Journal of clinical ultrasound, 2014,42(3):150-155.

10. Chamié LP, Ribeiro DMFR, Caiado AHM, et al. Translabial US and Dynamic MR Imaging of the Pelvic Floor: Normal Anatomy and Dysfunction. Radiographics, 2018,38(1):287-308.

11. Altomare DF, Picciariello A, Memeo R, et al. Pelvic floor function following ventral rectopexy versus STARR in the treatment of obstructed defecation. Techniques in coloproctology, 2018,22(4):289294.

12. Jalalizadeh M, Alshiek J, Santoro GA, et al. Six-Year Experience in Teaching Pelvic Floor Ultrasonography Using Pelvic Floor Phantoms. Obstetrics and gynecology, 2018,132(2):337-344.

13. Stachowicz N, Stachowicz S, Morawska D, et al. Assessment of the angle between puborectal muscles in women with and without stress urinary incontinence in three-dimensional sonograph. Prog Urol, 2014,67(4):447-452.

14. Murad-Regadas SM, Karbage SA, Bezerra LS, et al. Dynamic translabial ultrasound versus echodefecography combined with the endovaginal approach to assess pelvic floor dysfunctions: How effective are these techniques? Techniques in coloproctology, 2017,21(7):555-565.

15. Singh K, Reid WM, Berger LA. Magnetic resonance imaging of normal levator ani anatomy and function. Obstet Gynecol, 2002,99(3):433-438. 
16. Dietz HP, Abbu A, Shek KL. The levator-urethra gap measurement: a more objective means of determining levator avulsion? Ultrasound Obstet Gynecol, 2008,32(7):941-945.

17. Shobeiri SA, Rostaminia G, White D. The determinants of minimal levator hiatus and their relationship to the puborectalis muscle and the levator plate. BJOG-INT J OBSTET GY, 2013,120(2):205-211.

18. Brandão S, Parente M, Silva E, et al. Pubovisceralis Muscle Fiber Architecture Determination: Comparison Between Biomechanical Modeling and Diffusion Tensor Imaging. Annals of biomedical engineering, 2017,45(5):1255-1265.

19. Roos JE, Weishaupt D, Wildermuth $S$, et al. Experience of 4 years with open MR defecography: pictorial review of anorectal anatomy and disease. Radiographics, 2002,22(4):817-832.

20. Oliveira DA, Parente MPL, Calvo B, et al. The management of episiotomy technique and its effect on pelvic floor muscles during a malposition childbirth. Computer methods in biomechanics and biomedical engineering, 2017,20(11):1249-1259.

21. Shek KL, Dietz HP. Intrapartum risk factors of levator trauma. BJOG-INT J OBSTET GY, 2010, 117(12):1485-1492.

22. Chan SS, Cheung RY, Yiu AK, et al. Prevalence of levator ani muscle injury in Chinese women after first delivery. Ultrasound Obstet Gynecol, 2012,39(6):704-709.

23. Aydın S, Tuncel MA, Aydın SA, et al. Do we protect the pelvic floor with non-elective cesarean? A study of 3-D/4-D pelvic floor ultrasound immediately after delivery. Journal of obstetrics and gynaecology research, 2014,40(4):1037-1045.

24. Li R, Song Y, Ma M. Relationship between levator ani and bony pelvis morphology and clinical grade of prolapse in women. Clin Anat, 2015,28(6):813-819.

\section{Tables}

Due to technical limitations, all Table(s) are only available as a download in the supplemental files section.

\section{Figures}

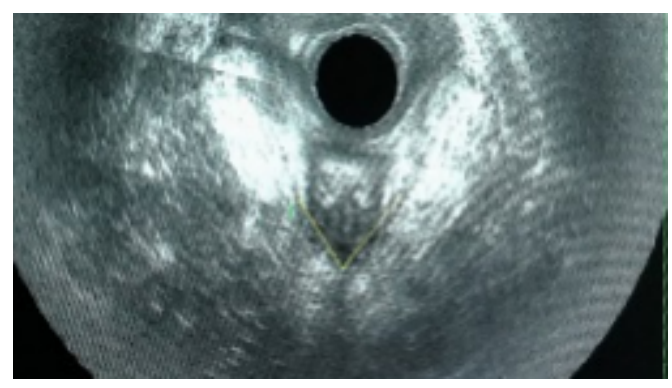

Figure 1 
The puborectalis angle ("V")

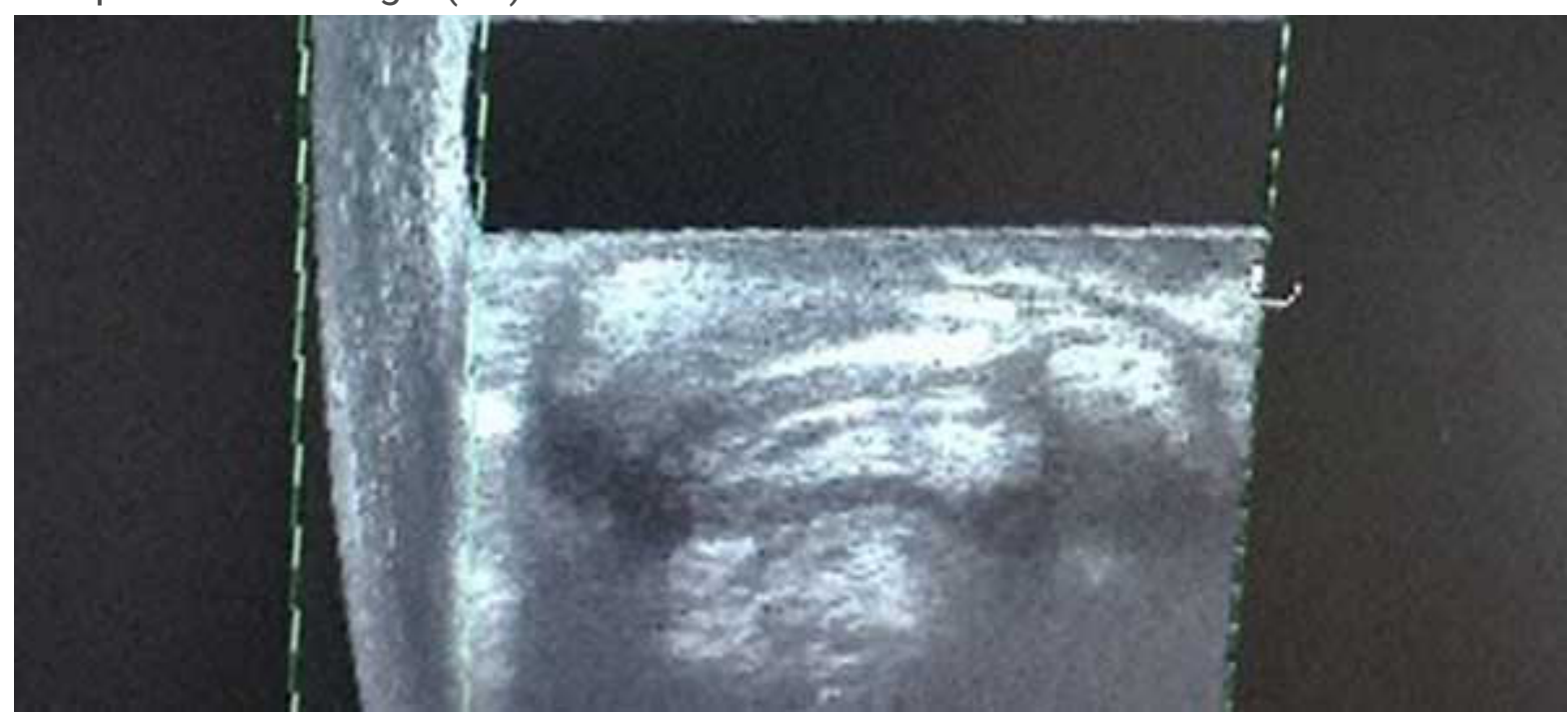

Figure 2

The puborectalis thickness at the 6 o'clock position with the patient in the lithotomy position ("]")

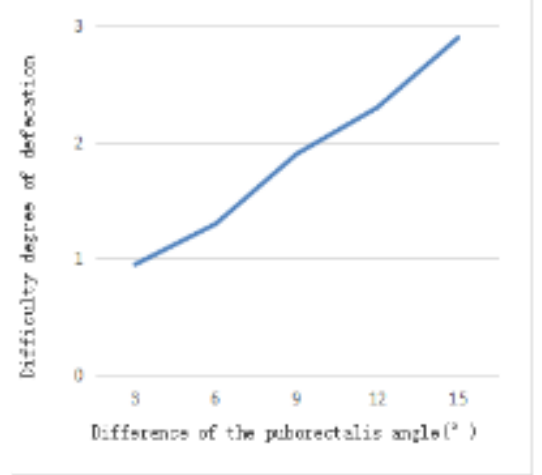

Figure 3

Correlation between the difference in the puborectalis angle and defecation difficulty

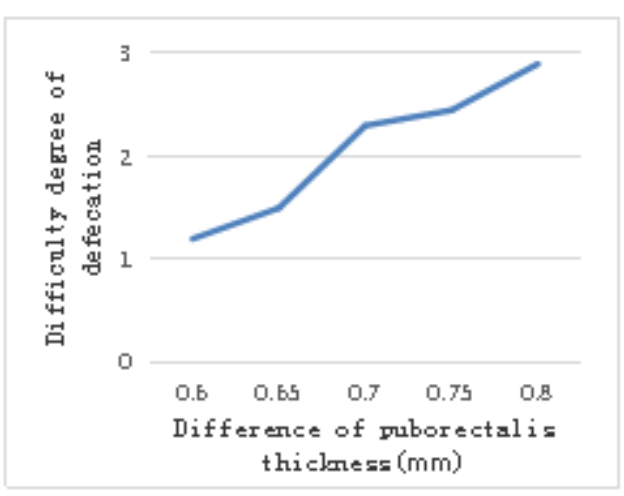

Figure 4 
Correlation between the difference in puborectalis thickness at the 6 o'clock position and defecation difficulty (Difficulty degree of defecation: 0 , natural defecation; 1 , defecation with less straining; 2 , defecation with more straining; 3 , defecation with external assistance.)

\section{Supplementary Files}

This is a list of supplementary files associated with this preprint. Click to download.

- Table1.jpg

- Table2.jpg 